\title{
Challenges, Coping Strategies, and Social Support among Breast Cancer Patients in Ghana
}

\author{
Ruth Boatemaa Benson, ${ }^{1}$ Bernice Cobbold, ${ }^{1}$ Ellen Opoku Boamah, \\ Cynthia Pomaa Akuoko $\left(\mathbb{C}^{3},{ }^{3}\right.$ and Daniel Boateng $\mathbb{C}^{4}$ \\ ${ }^{1}$ Department of Nursing, Garden City University College, Kumasi, Ghana \\ ${ }^{2}$ SDA Nursing and Midwifery Training College, Axim, Ghana \\ ${ }^{3}$ Christian Service University College, Kumasi, Ghana \\ ${ }^{4}$ School of Public Health, Kwame Nkrumah University of Science and Technology, Kumasi, Ghana \\ Correspondence should be addressed to Daniel Boateng; kingdannie@gmail.com
}

Received 19 June 2019; Revised 10 January 2020; Accepted 21 January 2020; Published 25 February 2020

Academic Editor: Carol J. Burns

Copyright $(2020$ Ruth Boatemaa Benson et al. This is an open access article distributed under the Creative Commons Attribution License, which permits unrestricted use, distribution, and reproduction in any medium, provided the original work is properly cited.

\begin{abstract}
Background. Despite the high incidence and mortality rate of breast cancer (BC) in Ghana, little attention has been given to the issue of how adult women cope with having BC. The aim of this study was to explore the challenges, coping strategies, and support systems among women diagnosed with BC in Ghana. Methods. A descriptive cross-sectional study was conducted from February to August 2017 at the Komfo Anokye Teaching Hospital (KATH), Ghana. A systematic random sampling technique was used to select 202 women with a confirmed diagnosis of BC. Coping strategies of women with BC were assessed using the Brief-COPE. The associations between sociodemographic characteristics, social network/support, and coping strategies were assessed using linear regression models. Results. The most and least adopted active coping strategies were religious coping and humors, respectively. Self-distraction and substance use were the most and least adopted avoidant coping strategies, respectively. Spouses and children offered the most support to women with BC; having support from 5 or more sources was associated with higher mean active coping (beta $[\beta] 1.14 ; 95 \%$ CI 0.66 to 1.62 ) and avoidant coping ( $\beta 1.46 ; 95 \%$ CI 0.98 to 1.94 ), as compared with having $<2$ sources of social support. Conclusion. This study demonstrates that women diagnosed with BC in Ghana adopt varied coping strategies to deal with these challenges. The forms of coping strategies adopted by women diagnosed with $\mathrm{BC}$ are influenced by the extent of social support received. Psychosocial counseling and support should be an integral part of BC management. Exploring and including social networks could play an important role in the management of BC in Ghana.
\end{abstract}

\section{Background}

Globally, breast cancer (BC) remains the second leading cause of mortality among women, with increasing rates particularly in developing countries where the majority of cases are diagnosed in late stages [1]. In Africa, it is the most commonly diagnosed cancer annually, with an estimated incidence of 168,690 cases and 74,072 related deaths in 2018 $[2,3]$. In Western Africa, although precise estimates are lacking as a result of absence of a cancer registry in most countries, recent GLOBOCAN data estimate age-standardized incidence and mortality rates of 37.3 and 17.8 per
100,000 female, respectively, in 2018 [2, 3], making it the most commonly diagnosed cancer in women, a shift from previous decades in which cervical cancer was the most commonly diagnosed cancer [4]. Survival from BC is known to be markedly lower in Africa compared with other regions in the world [5]. This is not different in Ghana where BC has become the leading cause of mortality among women and it is also the common cause of hospital admissions among Ghanaian women $[3,6]$.

Breast cancer diagnosis along with its treatment can contribute to physical, social, and psychological turmoil. These challenges extend to the periods of posttreatment and 
recovery. The psychosocial challenge in $\mathrm{BC}$ patients is related to the essence of the breasts in a woman's body image or femininity, sexuality, and motherhood [7]. Additional concerns include physical appearance and disfigurement after treatment, uncertainty regarding recurrence in the future, periods of anxiety and depression, difficulty maintaining hope, fear of death, as well as loss of self-esteem [8] for the patients and their loved ones, particularly life partners $[9,10]$. Challenges also include the shock of a cancer diagnosis and fears about the future or the side effects of treatment, such as nausea and fatigue [11]. Practical aspects of treatment such as the cost and travel also pose challenges [11]. Although relatively rare, BC presents with even more challenges in younger women, who tend to have a more aggressive biology and an associated poorer diagnosis [12]. A study conducted among BC patients in Ghana reported a high prevalence of depression (84.2\%) and anxiety (92.5\%), with $44 \%$ having both and with the prevalence being significantly higher among those living without partners [13]. Experiences of emotional reactions such as sadness, fear, severe pain leading to suicidal ideations, and loss of hope have also been reported among Ghanaian women diagnosed with $\mathrm{BC}$ [14].

Women diagnosed with $\mathrm{BC}$ develop coping strategies to deal with the multifactorial unpleasant experience of a psychological, social, and spiritual nature of their new life situation [15]. Coping is defined as current cognitive and/or behavioral efforts adopted to deal with specific external and/or internal stresses or anxieties that are valued as challenges and beyond the control and resources of the person [16]. Effective coping strategies are indispensable for adaptation and adjustment to $\mathrm{BC}$ and improvement in survival $[17,18]$. Coping strategies assist patients to deal with the problem that is causing the distress (problem-focused coping) and/or regulating stressful emotions (emotion-focused coping) [19, 20]. In Ghana, accepting the disease and surrendering to God has been reported as a coping strategy among women living with advanced BC [14]. The effectiveness of the coping strategy, however, depends on the degree of distress, variations in individual coping, the level of social support available and, to a large extent, the consultation skills and support of health professionals [21]. A link between social support and coping skills has been previously reported [22]. Early identification of coping strategies adopted by women with $\mathrm{BC}$ as well as patients who are coping poorly is important for treatment compliance, control of distress, and patient care in general.

Effective social support for BC patients could help reduce the negative impact of diagnosis and treatment and promote their psychological well-being $[23,24]$. The social networks include friends, family members, healthcare professionals, and neighbors, and forms of support are mainly instrumental, structural and functional, emotional, and informational [11]. Health workers play an important role in orienting patients prior to treatment to help them choose the most suitable methods, thereby minimizing the level of stress and uncertainty [25]. In a study among young women diagnosed with BC, an increase in social support (measured by amount of social contacts) was associated with increased likelihood of survival [22]. Women living with BC in Ghana are known to receive some form of support from their families, spouses, friends, health professionals, and spiritual leaders [14].

Despite the high incidence-mortality rate of $\mathrm{BC}$ in Ghana, and the related psychosocial challenges, less attention has been given to the issue of how patients cope with their condition.

1.1. Theoretical Framework. The theoretical framework for this study consists of perspectives on coping and social support previously applied in breast cancer research as reflected in Lazarus' [26] theory of appraisal, stress, and coping. According to Lazarus and Folkman [27, 28], coping involves persistently changing cognitive and behavioral efforts in order to manage specific external and/or internal demands that are seen as taxing or exceeding one's resources. The stress associated with a situation depends on an individual's appraisal of the situation and the behaviors directed at managing the stress. Lazarus [26] identified two major types of coping strategies. (i) Problem-focused coping lifestyle, which aims at solving the problem or situations, and (ii) Emotion-focused/ avoidant coping lifestyle, aimed at regulating the emotions related to stress. Problem-focused coping includes obtaining relevant information about what to do, whereas emotionfocused includes avoiding to think about the threat or reappraising it without changing the realities of stressful situations. The two coping strategies can both facilitate and impede each other throughout a stressful situation.

The concept of social support has been defined and operationalized in different ways and is identified as important in adjusting to breast cancer [29]. It is a multidimensional concept that is generally theorized from a quantitative-structural perspective of social networks, such as numbers of persons and formal relationships with them, or from a qualitative-functional perspective of social support, such as the perceived content and availability of relationships with significant others [30]. The qualitativefunctional support relates to the quality or function served by the structural support components and is mainly divided into the provision of instrumental, emotional, and informational support [31]. This study dwells on the buffer theory, which reflects the belief that support buffers against the adverse effects of stressors under conditions of high stress. The "buffering" effect of social support during stressful times with breast cancer has been previously investigated [32]. These studies generally conclude that social support from family and friends is associated with a better adjustment to $\mathrm{BC}$.

The aim of this study was to explore the challenges, coping strategies, and support systems reported by women diagnosed with $\mathrm{BC}$ in the Kumasi metropolis, Ghana, and to assess the associations among sociodemographic characteristics, social support, and coping strategies.

\section{Methods}

2.1. Study Design and Setting. The study was a descriptive cross-sectional design and was conducted at the oncology 
unit of Komfo Anokye Teaching Hospital (KATH), Kumasi, from February to August 2017. KATH is the second largest hospital in Ghana and the only tertiary health institution in the Ashanti Region. The oncology unit, which is part of the clinical directorate of the hospital, was opened in 2004.

2.2. Study Population and Sampling. The study population included women with a confirmed diagnosis of BC at the oncology unit of Komfo Anokye Teaching Hospital and was willing to participate in the study. The sample size was estimated using Cochran's sample size formula [33]: $n_{0}=\left(t^{2} *(p)(q) / d^{2}\right)$, where $n_{0}=$ sample size for a population; $t=$ standard normal deviation $=1.96 ; p=$ the estimated proportion of an attribute that is present in the population (assumed to be 15\%); $q=1-p(85 \%)$; and $d=$ degree of accuracy, set at $5 \%$ and a confident interval of $95 \%$, giving a total sample, $n$, of 196. 202 participants were finally included.

A pilot study was carried out first to establish the validity and reliability of the instruments and to assess the respondents' ability to accurately understand questions asked. The researchers were trained to ensure consistency in the administration of the questionnaire including the translation of the questions to the local language for participants. The questionnaire was administered by the researchers to all those selected for the study.

The study participants were recruited using a systematic random sampling technique. Prior to data collection, we estimated the average respondents needed per day using the estimated number of days for data collection and the average patients' attendance at the facility per day. We then estimated the sampling interval, $K$, using the average patients attendance and the average respondents needed per day ( $K=$ average patient attendance/needed respondents per day). On each day, during the visit hours, a first participant was identified and interviewed as the starting point, followed by the $K$ th respondent. This was repeated until the required sample size for each day was attained. We included women, 18 years and above, who had confirmed BC diagnosis at KATH and consented to participate in the study.

\subsection{Assessment of Coping Strategies and Challenges.} Coping strategies of women with $\mathrm{BC}$ were assessed using the Brief-COPE, which comprises 14 items, measuring two coping styles [34]. This instrument has been used to assess both habitual and dispositional coping (how people react in general), and more specific coping (how people react in relation to a specified stressful encounter). The Brief-COPE is a well validated scale that has been used extensively to assess coping strategies for many conditions including cancer [35] and depression [36].

The 14 items of the Brief-Cope measure self-distraction, active coping, denial, substance use, use of emotional support, use of instrumental support, behavioral disengagement, venting, positive reframing, planning, humor, acceptance, religion, and self-blame. The 14 items were grouped into two factors: adaptive/active coping (active coping, emotional support, positive reframing, planning, acceptance, religion, humor, and instrumental support) and maladaptive/avoidant subscale (venting, self-distraction, denial, substance use, behavioral engagement, and selfblaming) following Meyer [37]. The responses were on a Likert scale of four (4), directed at teasing out the frequency with which the women engaged in each strategy: 1 (I have not been doing this at all), 2 (I have been doing this a little bit), 3 (I have been doing this a medium amount), and 4 (I have been doing that a lot).

Challenges experienced were assessed on a Likert scale of five responses: 1 (never), 2 (rarely), 3 (sometimes), 4 (often), and 5 (always). There was a total of 10 items to measure possible challenges faced by women, including physical symptoms such as fatigue, fear of recurrence, body image disruption, sexual dysfunction, depression, and anxiety. The reliability statistics were mean 32.74 ; variance 67.75 ; standard deviation (SD) 8.23, and Cronbach's Alpha 0.86.

2.4. Other Variables. Sociodemographic characteristics and social support were assessed using a structured questionnaire. The sources of social support assessed were spouses, children, parents, religious bodies, friends, physician or caregivers, and support groups. This was developed with recourse to other studies [38]. Using a Likert scale of five (5) -1 (strongly disagree), 2 (disagree), 3 (neutral), 4 (agree), and 5 (strongly agree)-responses indicated the level of agreement to whether support is received from any of these sources. The reliability statistics for this scale were mean 23.91; SD 7.05, and Cronbach's Alpha 0.78. The form of support received from these sources was also assessed by responding "Yes" or "No" to each of the support systems. The forms of support assessed were financial, escort, emotional, and psychological counseling. Psychological support in this study meant psychological counseling to help the women deal with emotional and mental issues, whereas emotional support has to do with the receiving genuine concern and empathy from another person. The sources of social support were used as a proxy for the social network. This ranged from 1 to 8 , based on the 8 sources of social support described above. The social network was further grouped into $\leq 2,2-5$, and $\geq 5$ and used in the regression analysis to assess the influence of social networks on coping strategies.

The socioeconomic characteristics assessed were age, level of education (categorized as no education, primary, Junior High School, Senior High School, and Tertiary), marital status (categorized as single, married and living with partner, married and not living with partner, divorced/ widowed), religion (Christians, Muslims, and traditionalists or other religion), number of children, employment status, and type of occupation.

2.5. Statistical Analysis. All statistical analyses were carried out using SPSS, version 22 [39]. General characteristics are summarized as proportions and mean (SD) or median (25th and 75th percentiles) based on the distribution of the variables. The influence of sociodemographic characteristics and social network/support on coping strategies was assessed using linear regression models. Two models were 
fitted; model 1 to control for the sociodemographic characteristics, while model 2 adjusted for both the sociodemographic characteristics and social networks. Two-tailed significance tests were used throughout, and $p<0.05$ was considered statistically significant.

2.6. Ethical Considerations. An ethical approval and registration certificate was obtained from KATH to carry out the study. All study protocols were reviewed and approved by the Institutional Review Board of the Kwame Nkrumah University of Science and Technology-Committee for Human Research Publications and Ethics (CHRPE). Research procedures were explained, and informed consent was received from all participants.

\section{Results}

3.1. Sociodemographic Characteristics of Study Respondents. The mean age (SD) of the women was 55 years (13.4), and age ranged from 33 to 87 years; $27.2 \%$ of the women had no formal education, while $17.8 \%$ had tertiary education. Majority of the women were married, of which $39.6 \%$ were living with their partners. The majority $(68.8 \%)$ were Christians. The median (25th, 75th percentile) number of children was 3 (2, 5 ), and $10.4 \%$ had no children. The majority $(68.8 \%)$ were employed with $40.3 \%$ being traders (Table 1 ).

3.2. Experience of Challenges. The mean responses indicate that most of the women sometimes experienced physical symptoms such as fatigue, trouble with sleeping, pain, and burnout, mean, sexual dysfunction, threat to their safety, and felt vulnerable. Most of the women often had intrusive thoughts about their illness and hence were depressed and anxious. Some women also had communication issues with their partners and body image disruptions (Table 2).

3.3. Support Systems for Women Diagnosed with Breast Cancer. The highest form of support was from religious bodies, churches, and the community (mean $=4.01$ ) followed by children (mean $=3.67)$. The least form of support was from support groups $($ mean $=2.26$ ) (Figure 1$)$. We further aggregated the sources of social support and used as a proxy for the social network. The results indicate that approximately half (51\%) of the women had support from five (5) sources or more, whereas $11.4 \%$ had support from two (2) sources or fewer. $10.9 \%, 8.9 \%$, and $11.9 \%$ had support from six (6), seven (7), and eight (8) sources, respectively.

The forms of support received by the women were financial, escort, emotional, and psychological or counseling (Figure 2). The most form of support offered by spouses or children was financial $(78.7 \%)$ followed by the escort $(62.4 \%)$. The most form of support offered by religious groups was emotional support (57.4\%). Psychological support or counseling was also provided mostly by physicians and care providers (74.8\%). On the average, majority, $57 \%$ of the women had only one form of support, whereas $31 \%$,
$7.7 \%$, and $4.2 \%$ had two, three, and four forms of support, respectively.

3.4. Coping Strategies. Majority of women frequently engaged in active coping strategies. The most adopted active coping strategy was religious coping, while the least adopted was humor. Self-distraction and substance use were the most and least adopted avoidant coping strategies, respectively (Table 3).

3.5. Factors Influencing Coping Strategies. Age, marital status, and employment status were associated with coping strategies adopted by women with BC. Higher age has no influence on active coping but might be associated with less avoidant coping (only shown in model 1). Being married was also associated with higher active coping in model 1 but not with avoidant coping. Being employed had a positive association with both active and avoidant coping strategies, although effect on avoidant coping was only observed in model 1 . In the adjusted model, being employed was associated with 0.38 increase in mean active coping, as compared with those not employed ( $\beta 0.38 ; 95 \%$ CI $0.06,0.70)$. Support by more than 2 persons increases both active and avoidant coping. Women who had more than 5 sources of support had $1.41(\beta 1.14 ; 95 \%$ CI $0.66,1.62)$ and $1.46(\beta 1.46$; 95\% CI $0.98,1.94)$ higher mean active and avoidant coping, respectively, compared with those who had support from $<2$ sources. Similarly, higher mean active and avoidant coping was observed for women with 2 to 5 sources of social support compared with those with $<2$ sources (Table 4).

\section{Discussion}

The women often had unpleasant thoughts about their illness, including fear of recurrence of the disease and were anxious and depressed. Sometimes, the women felt vulnerable and had sexual dysfunction, physical symptoms such as fatigue, burnout, and trouble with sleeping, and partner communication issues. We also found that coping strategies were associated with the extent of social network or support as well as the employment status of women diagnosed with BC.

Our findings corroborates those in Ghana that found a high prevalence of depression and anxiety [13] and experiences of sadness, fear, and severe pain leading to suicidal ideas among women living with BC [14]. This is also consistent to the reports from women elsewhere who expressed fear and anxiety and harbored fears of the recurrence of the disease [40] and disruption of body image [41]. Understanding these challenges and the nature of anxieties in cancer patient populations is important as abnormal anxiety affects the psychological well-being of the patients [42].

These intrusive thoughts, fear, and anxiety are partly related to the perceived stigma associated with BC diagnosis, especially in these settings. Cancer stigma is present in many communities, and it is a feared outcome of a cancer diagnosis, thereby militating against screening, early diagnosis, and treatment seeking for women with symptoms [43]. Losing one breast as a result of surgery, for instance, could 
TABLE 1: Background characteristics of respondents.

\begin{tabular}{|c|c|c|}
\hline Variables & $\begin{array}{c}\text { Frequency } \\
N=202\end{array}$ & Percentage \\
\hline Age, mean \pm SD & $55 \pm 13.4$ & \\
\hline$<40$ & 23 & 11.4 \\
\hline $40-50$ & 71 & 35.1 \\
\hline $50-60$ & 48 & 23.8 \\
\hline$>60$ & 60 & 29.7 \\
\hline \multicolumn{3}{|l|}{ Education } \\
\hline No formal & 55 & 27.2 \\
\hline Primary school & 54 & 26.7 \\
\hline Junior high or middle school & 36 & 17.8 \\
\hline Senior high or secondary school & 21 & 10.4 \\
\hline Tertiary & 36 & 17.8 \\
\hline \multicolumn{3}{|l|}{ Marital status } \\
\hline Single & 27 & 13.4 \\
\hline Married and living with partner & 80 & 39.6 \\
\hline Married and not living with partner & 40 & 19.8 \\
\hline Divorced/widowed & 55 & 27.2 \\
\hline \multicolumn{3}{|l|}{ Religion } \\
\hline Christians & 139 & 68.8 \\
\hline Muslims & 61 & 30.2 \\
\hline No religion/traditional & 2 & 1.0 \\
\hline Number of children, median (25th, 75 th percentile) & $3(2,5)$ & \\
\hline None & 21 & 10.4 \\
\hline $1-3$ & 84 & 41.6 \\
\hline $4-6$ & 79 & 39.1 \\
\hline 7 or more & 18 & 8.9 \\
\hline \multicolumn{3}{|l|}{ Employment status } \\
\hline Employed & 139 & 68.8 \\
\hline Unemployed & 63 & 31.2 \\
\hline \multicolumn{3}{|l|}{ Types of occupation $(n=139)$} \\
\hline Civil or public servant & 20 & 14.4 \\
\hline Health professional & 11 & 7.9 \\
\hline Farmer & 40 & 18.8 \\
\hline Trader & 56 & 40.3 \\
\hline Artisan & 12 & 8.6 \\
\hline
\end{tabular}

$\mathrm{SD}$, standard deviation.

TABLE 2: Experience of challenges among women diagnosed with breast cancer.

\begin{tabular}{|c|c|c|c|}
\hline Responses/items & Mean & SD & SR \\
\hline Physical symptoms such as fatigue, trouble sleeping, or pain, weight gain, burnout & 3.35 & 1.01 & Sometimes \\
\hline Fear of recurrence & 3.73 & 1.20 & Often \\
\hline Feelings of vulnerability & 3.21 & 1.14 & Sometimes \\
\hline Body image disruption & 3.36 & 1.33 & Sometimes \\
\hline Intrusive thoughts about illness & 3.68 & 1.09 & Often \\
\hline Marital/partner communication issues & 2.58 & 1.41 & Sometimes \\
\hline Sexual dysfunction & 2.71 & 1.41 & Sometimes \\
\hline Experience of threats to safety & 3.22 & 1.21 & Sometimes \\
\hline Reduced sexual interest and decreased sexual function. & 2.80 & 1.39 & Sometimes \\
\hline Depression and anxiety & 3.95 & 1.15 & Often \\
\hline
\end{tabular}

SR, summary response; SD, standard deviation. Responses: $1=$ never; $2=$ rarely; $3=$ sometimes; $4=$ often; $5=$ always. Challenges faced by women diagnosed with breast cancer measured using a questionnaire of 10 items, that is, possible challenges faced.

lead to stigma and loss of self-esteem, resulting in distress and pain [44]. In Ghana, stigmatization and cultural barriers are important elements in the fight against BC. BC is largely seen as a spiritual disease, as a curse on a family (especially those with positive family history), caused by witchcraft, and as an incurable disease. This perception is similar among both urban and rural Ghanaian women [45, 46]. These perceptions, beliefs, and traditions impact on disease management and health-seeking behavior in relation to BC [43]. It is important to assist these women to adjust to these changes and adopt strategies to cope and deal with their current situation. 


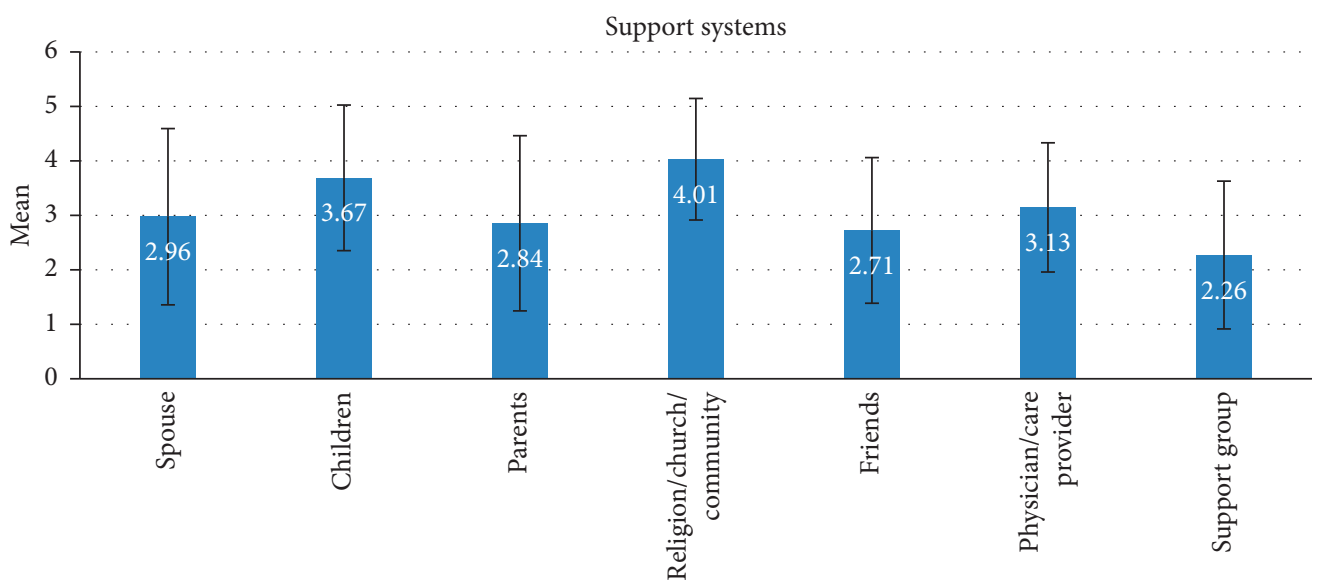

FIGURE 1: Support systems in coping with breast cancer. Error bars = standard deviation; response: $1=$ strongly disagree; $2=$ disagree; $3=$ neutral; 4 = agree; 5 = strongly agree.



FIGURE 2: Forms of support received by women diagnosed with breast cancer.

Although sexual dysfunction is well acknowledged as a major challenge among women diagnosed with $\mathrm{BC}$, this was least reported in this study. Receiving a BC diagnosis and undergoing associated treatments including long-term therapy can impair sexual function via a number of mechanisms, including disrupting ovarian function, body image, intimacy, and relationships [47], contributing to a lower quality of life in BC survivorship. The low prevalence of sexually related challenges in this study could be due to underreporting. A population-based study among Ghanaian women reported an overall prevalence of $45.6 \%$ of female sexual dysfunction (FSD) [48]. High levels of environmental sexual reproductive health stigma among young women in Ghana have also been reported [49]. Majority of respondents in this study may also not be exposed to the various clinical factors such as hormonal alterations induced by chemotherapy and radiotherapy or physiological and functional disturbances related to the deterioration of physical condition. They might also be resilient to other psychological factors that induce disturbances in sexual behavior such as anxious/depressive reactions in adapting to the illness and treatment, hypochondriacal and cancer phobic reactions, regressive tendencies, and loss of self-esteem [50].
In an attempt to deal with their health and psychosocial issues relating to the diagnosis of $\mathrm{BC}$, these women adopt various coping strategies. We found a high frequency of use of active coping strategies among the women studied. These strategies are adaptive, favorable, and problem-focused, and individuals accept and actively attempt to deal with their situation. The women in this study engaged frequently in positive reframing, planning, acceptance, and religious coping. This corroborates previous findings, which identified religion, acceptance, planning, active coping, and positive reframing as various active coping strategies among women diagnosed with BC in Iran [22]. These strategies have been shown to have a positive influence on the women's adjustment to $\mathrm{BC}$, by influencing positively the psychological well-being and health behaviors of these patients [51].

Religion was the most frequently used active coping strategy in this study. This corroborates previous findings in Ghana that suggested that women diagnosed with advanced $\mathrm{BC}$ surrendered to God and prayed for the will of God to prevail regarding their conditions [14]. Findings from other settings also found a religious approach and spiritual fighting as key active coping strategies among newly diagnosed cancer patients [52]. These studies suggest that a 
TABLE 3: Mean and standard deviation of coping strategies of women diagnosed with breast cancer.

\begin{tabular}{lcc}
\hline Variables & Mean $^{\mathrm{a}}$ & $\mathrm{SD}$ \\
\hline Active/problem focus (adaptive) coping strategies & \\
Active coping & 5.84 & 0.92 \\
Emotional support & 6.03 & 0.91 \\
Positive reframing & 5.18 & 0.90 \\
Planning & 5.71 & 0.99 \\
Acceptance & 4.80 & 0.87 \\
Religious coping & 6.97 & 0.78 \\
Humor & 3.97 & 1.04 \\
Instrumental support & 5.89 & 0.89 \\
Overall mean & 5.55 & 1.06 \\
Avoidant/emotional focused (maladaptive) coping strategies \\
Venting/voicing & 5.39 & 0.91 \\
Self-distraction & 5.45 & 0.94 \\
Denial & 5.07 & 1.18 \\
Substance use & 3.23 & 0.88 \\
Behavioral disengagement & 4.48 & 0.93 \\
Self-blaming & 3.76 & 0.91 \\
Overall mean & 4.56 & 1.06 \\
\hline
\end{tabular}

${ }^{\mathrm{a}}$ Computed by summing the means of two items/questions for each cooping variables. SD, standard deviation. Responses: $1=\mathrm{I}$ have not been doing this at all; 2 = I've been doing this a little bit; $3=$ I've been doing this a medium amount; 4 = I've been doing this a lot. Coping strategies of women with BC were assessed using the Brief-COPE, which comprises 14 items, measuring two coping styles [34].

relationship with God provides a consistent and reliable source of support and comfort as well as support from a religious community, especially among people of African descent [53]. In Ghana, women demonstrate their will to live by praying positively and being hopeful for long life despite their predicament [14]. In a study conducted in the United Kingdom, a majority of the $\mathrm{BC}$ patients used positive religious coping to some degree at surgery [54]. Participating in religious service ignites a sense of belonging among these women and buffers against feelings of isolation [55].

This study also assessed avoidant coping strategies among women. These strategies are problem focused and include strategies such as denial, behavioral disengagement, and alcohol/drug. We found that venting or voicing, selfdistraction, and behavioral disengagement were frequently used avoidant coping strategies, whereas humor, substance use, and self-blaming were less frequently used. It is important to assess how these strategies influence health outcomes among this group of patients. The influence of coping on psychological well-being depends on the situation and characteristics of the individual [56]. Avoidant coping would have less of a negative association with patients' mental and physical well-being when patient symptoms were low and vice versa [57].

Social support networks have been shown to be significantly important in helping people manage life crises and health challenges [58]. The sources of support endorsed in this study were spouses, children, parents, religious bodies or community, friends and health providers, with children being the most cited. Similarly, a study conducted in Ghana found that $\mathrm{BC}$ patients received support from their families, spouses, friends, health professionals, and spiritual leaders
[14]. Spouses, children, families, and friends were dominant in providing forms of support to these women. This corroborates findings from previous studies, which showed that spouses and families were supportive, giving confidence, listening to the patients, and supporting them in the decision-making process about the treatment $[59,60]$. A recent systematic review also found emotional support by spouses, family members, and friends as the main forms of support for women with BC [61]. Religious bodies provided emotional support, psychological counseling, and some form of financial assistance, whereas physicians and care providers provided counseling and emotional support to these women. Arora et al. [60] also found that most women diagnosed with $\mathrm{BC}$ receive helpful informational and emotional support from healthcare providers. These supports are important for the psychological well-being of the patients especially in the early stages of diagnosis, where patients face the greatest anxiety [62], and the absence of it could hamper effective healthcare access by BC patients [63]. Support from support groups was less in this study compared with other sources of support. It is important to assess the formats of these support groups, costs involved in attending to these groups as well as the contents of these groups and how these influences assess, and provision of support to BC patients in Ghana.

We found an association between support systems and coping strategies among women diagnosed with BC. Women with more sources of social support were more likely to adopt a coping strategy as compared with those with fewer sources of social support. Being employed, and therefore having more social contact, was also positively associated with active coping strategy among the women studied. This corroborates previous evidence, which shows that an increase in social support increases women's survival by enhancing their coping skills, providing emotional support, and expanding opportunities for information sharing [64]. Women with BC who are in support groups have significantly better psychological well-being, more posttraumatic growth, better self-image, better sexual functioning and higher sexual satisfaction, better coping and communication, and fewer physical symptoms associated with BC [65]. A meta-analysis of existing literature on interventions delivered to family caregivers of cancer patients also showed a significant, positive effect on multiple health outcomes [66].

Contrary to previous findings, social support was positively associated with avoidant coping strategies in this study, indicating that although social support encourages women to adopt active coping strategies, some women still resort to avoidant coping despite having support socially. This is supported by Lazarus and Rice [56] notion that avoidant coping strategies are not always maladaptive and might have differential effects depending on the degree of symptom and distress a patient is experiencing [56]. We also believe that the avoidant coping lifestyles are rooted in stigmatization and negative perceptions, which could not be demystified regardless of their support from their social networks. We recommend further studies into stigmatization, psychosocial challenges, and how these influence strategies with breast cancer. It is also important to further 


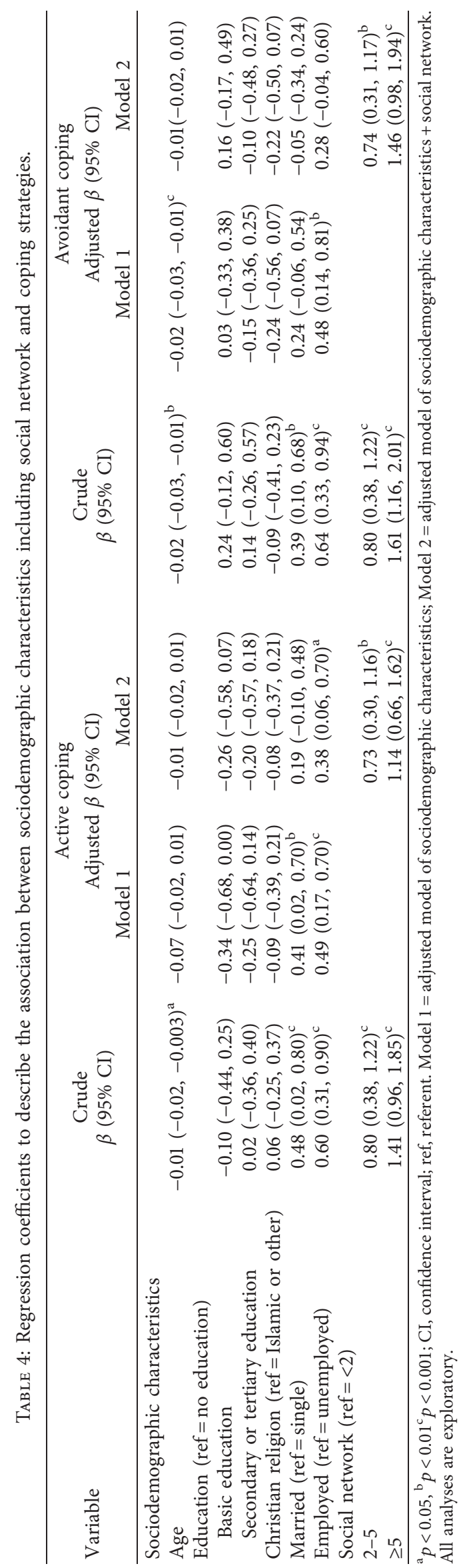


explore support systems available to these women at all levels and how they relate to survival outcomes and quality of life.

4.1. Clinical Implications. Findings from this study show various challenges associated with BC diagnosis in Ghana and point to the need for healthcare professionals to elicit these challenges and support the women with psychosocial support. This study further shows the need to elicit the coping strategies adopted among women diagnosed with $\mathrm{BC}$ and help identify women who require special counseling and support. Psychological screening is recommended for women diagnosed with breast cancer during their treatment period. Such screening can support healthcare professionals with information and better understanding of the psychosocial needs of breast cancer patients and the coping skills required to meet those needs.

4.2. Limitations. The instruments used to measure coping in this study had not been previously validated in this setting although they were pretested to ensure their reliability in this study population. Being a cross-sectional analysis, causation cannot be concluded. Previous studies have however identified social support as an important factor in managing challenges associated with BC diagnosis. This study also did not collect information on the clinical characteristics of the patients, including the stage of the disease and the kinds of treatment received by the patients in this study.

\section{Conclusion}

In conclusion, this study demonstrated varied challenges faced by women diagnosed with BC in Ghana, including fear and anxiety and intrusive thoughts about their illness. These women also most frequently used various active coping strategies, especially religious coping in dealing with their health and psychosocial issues. Women diagnosed with $\mathrm{BC}$ explore and receive various forms of support, and their extent of social support and social networks influences their coping with challenges associated with their ailment. It is important to ascertain the level of social support and further explore and create opportunities to involve family members in breast care management.

\section{Abbreviations:}

BC: $\quad$ Breast cancer

KATH: Komfo Anokye Teaching Hospital

KNUST: Kwame Nkrumah University of Science and Technology

SPSS: $\quad$ Statistical Package for Social Sciences

CHRPE: Committee for Human Research Publications and Ethics

SD: $\quad$ Standard deviation.

\section{Data Availability}

Data for this study are securely kept by the principal investigator and will be made available upon request.

\section{Conflicts of Interest}

The authors have no funding or conflicts of interest to disclose.

\section{Acknowledgments}

The authors are grateful to the staff and management of the Oncology Department of the Komfo Anokye Teaching Hospital, Ghana, for their assistance in this study and to all participants who took part in the study.

\section{References}

[1] World Health Organization, Breast Cancer: Prevention and Control, World Health Organization, Geneva, Switzerland, 2016, http://www.who.int/cancer/detection/breastcancer/en/ index1.html.

[2] J. Ferlay, M. Colombet, I. Soerjomataram et al., Global and Regional Estimates of the Incidence and Mortality for 38 Cancers, GLOBOCAN 2018, Brüssel, Belgien, 2018.

[3] F. Bray, J. Ferlay, I. Soerjomataram, R. L. Siegel, L. A. Torre, and A. Jemal, "Global cancer statistics 2018: globocan estimates of incidence and mortality worldwide for 36 cancers in 185 countries," A Cancer Journal for Clinicians, vol. 68, no. 6, pp. 394-424, 2018.

[4] The Cancer Atlas, Cancer in Sub-Saharan Africa, WHO, Geneva, Switzerland, 2017, http://canceratlas.cancer.org/theburden/cancer-in-sub-saharan-africa/.

[5] R. Sankaranarayanan, R. Swaminathan, H. Brenner et al., "Cancer survival in Africa, Asia, and Central America: a population-based study," The Lancet Oncology, vol. 11, no. 2, pp. 165-173, 2010.

[6] M. Ohene-Yeboah and E. Adjei, "Breast cancer in Kumasi, Ghana," Ghana Medical Journal, vol. 46, no. 1, pp. 8-13, 2012.

[7] J. Giese-Davis, K. Collie, K. M. S. Rancourt, E. Neri, H. C. Kraemer, and D. Spiegel, "Decrease in depression symptoms is associated with longer survival in patients with metastatic breast cancer: a secondary analysis," Journal of Clinical Oncology, vol. 29, no. 4, pp. 413-420, 2011.

[8] C. Bertero and M. Chamberlain Wilmoth, "Breast cancer diagnosis and its treatment affecting the self," Cancer Nursing, vol. 30, no. 3, pp. 194-202, 2007.

[9] H. Moreira and M. C. Canavarro, "Psychosocial adjustment and marital intimacy among partners of patients with breast cancer: a comparison study with partners of healthy women," Journal of Psychosocial Oncology, vol. 31, no. 3, pp. 282-304, 2013.

[10] T. Badger, C. Segrin, S. M. Dorros, P. Meek, and A. M. Lopez, "Depression and anxiety in women with breast cancer and their partners," Nursing Research, vol. 56, no. 1, pp. 44-53, 2007.

[11] Cancer Australia, "Living with breast cancer," 2017, https://www.cancer.org/cancer/breast-cancer/living-as-abreast-cancer-survivor.html.

[12] Oncology Nurse Advisor, "Managing breast cancer in younger women: challenges and solutions," 2016, http:// www.oncologynurseadvisor.com/breast-cancer/managingbreast-cancer-in-younger-women-challenges-and-solutions/ article/466474/.

[13] T. Calys, A. Senaedza, and L. Clegg, "Anxiety and depression among breast cancer patients in a Ghana," Postgraduate Medical Journal of Ghana, vol. 6, no. 1, pp. 54-58, 2017.

[14] A. B. Bonsu, L. Aziato, and J. N. A. Clegg-Lamptey, "Living with advanced breast cancer among Ghanaian women: emotional and psychosocial experiences," International 
Journal of Palliative Care, vol. 2014, Article ID 403473, 9 pages, 2014.

[15] K. Abrahamson, "Dealing with cancer-related distress," American Journal of Nursing, vol. 110, no. 4, pp. 67-69, 2010.

[16] R. S. Lazarus and S. Folkman, Stress, Appraisal, and Coping, vol. 116, Springer, Berlin, Germany, 1984, http://www. amazon.com/dp/0826141919.

[17] P. Reynods, S. Hurley, M. Torres, J. Jackson, P. Boyd, and V. W. Chen, "Use of coping strategies and breast cancer survival: results from the black/white cancer survival study," American Journal of Epidemiology, vol. 152, no. 10, pp. 940949, 2000.

[18] P. Kvillemo and R. Bränström, "Coping with breast cancer: a meta-analysis," PLoS One, vol. 9, no. 11, Article ID e112733, 2014.

[19] S. Folkman, R. S. Lazarus, C. Dunkel-Schetter, A. DeLongis, and R. J. Gruen, "Dynamics of a stressful encounter: cognitive appraisal, coping, and encounter outcomes," Journal of Personality and Social Psychology, vol. 50, no. 5, pp. 992-1003, 1986.

[20] S. Folkman, R. S. Lazarus, R. J. Gruen, and A. DeLongis, "Appraisal, coping, health status, and psychological symptoms," Journal of Personality and Social Psychology, vol. 50, no. 3, pp. 571-579, 1986.

[21] C. S. Carver and J. Connor-Smith, "Personality and coping," Annual Review of Psychology, vol. 61, no. 1, pp. 679-704, 2010.

[22] N. Khalili, Z. Farajzadegan, F. Mokarian, and F. Bahrami, "Coping strategies, quality of life and pain in women with breast cancer," Iranian Journal of Nursing and Midwifery Research, vol. 18, no. 2, pp. 105-111, 2013.

[23] S. Cohen and T. A. Wills, "Stress, social support, and the buffering hypothesis," Psychological Bulletin, vol. 98, no. 2, pp. 310-357, 1985.

[24] K. Ell, R. Nishimoto, L. Mediansky, J. Mantell, and M. Hamovitch, "Social relations, social support and survival among patients with cancer," Journal of Psychosomatic Research, vol. 36, no. 6, pp. 531-541, 1992.

[25] Z. Ng, M. Ong, T. Jegadeesan, S. Deng, and C. Yap, "Breast cancer: exploring the facts and holistic needs during and beyond treatment," Healthcare, vol. 5, no. 2, p. 26, 2017.

[26] R. Lazarus, Stress and Emotion: A New Synthesis, Springer Publishing Co., New York, NY, USA, 1999, http://psycnet.apa. org/record/1999-02362-000.

[27] A. Biggs, P. Brough, and S. Drummond, "Lazarus and Folkman's psychological stress and coping theory," in The Handbook of Stress and Health, pp. 349-364, John Wiley \& Sons, Hoboken, NJ, USA, 2017.

[28] R. S. Lazarus and S. Folkman, Stress, Appraisal, and Coping, Springer, Berlin, Germany, 1984, https://books.google.nl/books/ about/Stress_Appraisal_and_Coping.html?id=i-ySQQuUpr8 C\&redir_esc $=y$.

[29] K. Dukes Holland and C. K. Holaha, "The relation of social support and coping to positive adaptation to breast cancer," Psychology \& Health, vol. 18, no. 1, pp. 15-29, 2003.

[30] B. Nausheen, Y. Gidron, R. Peveler, and R. Moss-Morris, "Social support and cancer progression: a systematic review," Journal of Psychosomatic Research, vol. 67, no. 5, pp. 403-415, 2009.

[31] D. Finfgeld-Connett, "Clarification of social support," Journal of Nursing Scholarship: an official publication of Sigma Theta Tau International Honor Society of Nursing/Sigma Theta Tau, vol. 37, no. 1, pp. 4-9, 2005.

[32] T. M. Gremore, D. H. Baucom, L. S. Porter, J. S. Kirby, D. C. Atkins, and F. J. Keefe, "Stress buffering effects of daily spousal support on women's daily emotional and physical experiences in the context of breast cancer concerns," Health Psychology, vol. 30, no. 1, pp. 20-30, 2011.

[33] W. G. Cochran, Wiley Series in Probability and Mathematical Statistics Applied, Vol. 20, Wiley, Hoboken, NJ, USA, 1977.

[34] C. S. Carver, "You want to measure coping but your protocol' too long: consider the brief cope," International Journal of Behavioral Medicine, vol. 4, no. 1, pp. 92-100, 1997.

[35] MIDSS. Brief COPE, 2018, http://www.midss.org/content/ brief-cope.

[36] C. Cooper, C. Katona, M. Orrell, and G. Livingston, “Coping strategies, anxiety and depression in caregivers of people with Alzheimer's disease," International Journal of Geriatric Psychiatry, vol. 23, no. 9, pp. 929-936, 2008.

[37] B. Meyer, "Coping with severe mental illness: relations of the brief cope with symptoms, functioning, and well-being," Journal of Psychopathology and Behavioral Assessment, vol. 23, no. 4, pp. 265-277, 2001.

[38] C. H. Kroenke, M. L. Kwan, A. I. Neugut et al., "Social networks, social support mechanisms, and quality of life after breast cancer diagnosis," Breast Cancer Research and Treatment, vol. 139, no. 2, pp. 515-527, 2013.

[39] IBM Corp, IBM SPSS Statistics for Windows: Version 22.0, IBM Corp, Armonk, NY, USA, 2011.

[40] M. A. A. Doumit, N. El Saghir, H. Abu-Saad Huijer, J. H. Kelley, and N. Nassar, "Living with breast cancer, a Lebanese experience," European Journal of Oncology Nursing, vol. 14, no. 1, pp. 42-48, 2010.

[41] A. M. Shoma, M. H. Mohamed, N. Nouman et al., "Body image disturbance and surgical decision making in Egyptian post menopausal breast cancer patients," World Journal of Surgical Oncology, vol. 7, no. 1, 2009.

[42] S. M. S. Baqutayan, "The effect of anxiety on breast cancer patients," Indian Journal of Psychological Medicine, vol. 34, no. 2, pp. 119-123, 2012.

[43] C. P. Akuoko, A. Sapino, E. Armah et al., "Barriers to early presentation and diagnosis of breast cancer among African women living in sub-Saharan Africa," PLoS One, vol. 12, no. 2, Article ID e0171024, 2017.

[44] M. Al-Azri, H. Al-Awisi, S. Al-Rasbi et al., "Psychosocial impact of breast cancer diagnosis among Omani women," Oman Medical Journal, vol. 29, no. 6, pp. 437-444, 2014.

[45] A. Asobayire and R. Barley, "Women's cultural perceptions and attitudes towards breast cancer: northern Ghana," Health Promotion International, vol. 30, no. 3, pp. 647-57, 2015.

[46] S. A. Aheng, Stigmatization is a Hindrance to the Fight Against Breast Cancer in Ghana, ModenGhana, Accra, Ghana, 2015, https://www.modernghana.com/news/659540/1/stigmatization-isa-hindrance-to-the-fight-against-breast-cancer-in-ghana-d.html.

[47] S. Onen, M. Elbi, A. Oyan, and M. Alper, "Effects of surgery type on body image, sexuality, self-esteem, and marital adjustment in breast cancer: a controlled study," Turk Psikiyatr dergisi, vol. 15, no. 4, pp. 264-275, 2004.

[48] E. P. Imbeah, B. A. Afrane, I. A. Kretchy et al., "Prevalence and self-management of female sexual dysfunction among women in six regions of Ghana: a cross-sectional study," Journal of Women's Health, Issues and Care, vol. 4, no. 6, 2015.

[49] K. S. Hall, E. Morhe, A. Manu et al., "Factors associated with sexual and reproductive health stigma among adolescent girls in Ghana," PLoS One, vol. 13, no. 4, Article ID e0195163, 2018.

[50] D. Novick, W. Montgomery, J. Aguado et al., "Which somatic symptoms are associated with an unfavorable course in Asian patients with major depressive disorder?," Journal of Affective Disorders, vol. 149, no. 1-3, pp. 182-188, 2013. 
[51] J. Kim, J. Y. Han, B. Shaw, F. McTavish, and D. Gustafson, "The roles of social support and coping strategies in predicting breast cancer patients' emotional well-being: testing mediation and moderation models," Journal of Health Psychology, vol. 15, no. 4, pp. 543-552, 2010.

[52] F. Taleghani, Z. P. Yekta, and A. N. Nasrabadi, "Coping with breast cancer in newly diagnosed Iranian women," Journal of Advanced Nursing, vol. 54, no. 3, pp. 265-272, 2006.

[53] L. L. Roff, C. E. Simon, D. Nelson-Gardell, and H. M. Pleasants, "Spiritual support and African American breast cancer survivors," Affilia, vol. 24, no. 3, pp. 285-99, 2009.

[54] I. C. V. Thuné-Boyle, J. Stygall, M. R. S. Keshtgar, T. I. Davidson, and S. P. Newman, "Religious coping strategies in patients diagnosed with breast cancer in the UK," PsychoOncology, vol. 20, no. 7, pp. 771-782, 2011.

[55] L. Mcdougle, S. Konrath, M. Walk, and F. Handy, "Religious and secular coping strategies and mortality risk among older adults," Social Indicators Research, vol. 125, no. 2, pp. 677-94, 2015.

[56] R. Lazarus and V. Rice, "Evolution of a model of stress, coping, and discrete emotions," Handbook Stress Coping, pp. 195-222, Sage, Thousand Oaks, CA, USA, 2000.

[57] T. Kershaw, L. Northouse, C. Kritpracha, A. Schafenacker, and D. Mood, "Coping strategies and quality of life in women with advanced breast cancer and their family caregivers," Psychology \& Health, vol. 19, no. 2, pp. 139-55, 2004.

[58] L. J. Kristjanson and S. Aoun, "Palliative care for families: remembering the hidden patients," The Canadian Journal of Psychiatry, vol. 49, no. 6, pp. 359-365, 2004.

[59] M. Ardahan and O. U. Yesilbalkan, "Perceived family support of women with breast cancer and affecting factors in Turkey," Asian Pacific Journal of Cancer Prevention, vol. 11, no. 5, pp. 1425-1429, 2010.

[60] N. K. Arora, L. J. Finney Rutten, D. H. Gustafson, R. Moser, and R. P. Hawkins, "Perceived helpfulness and impact of social support provided by family, friends, and health care providers to women newly diagnosed with breast cancer," Psycho-Oncology, vol. 16, no. 5, pp. 474-486, 2007.

[61] A. F. Fernandes, A. Cruz, C. Moreira, M. C. Santos, T. Silva, and A. F. Fernandes, "Social support provided to women undergoing breast cancer treatment: a study review," $A d$ vances in Breast Cancer Research, vol. 3, no. 2, pp. 47-53, 2014.

[62] S. M. Silva, C. Crespo, and M. C. Canavarro, "Pathways for psychological adjustment in breast cancer: a longitudinal study on coping strategies and posttraumatic growth," Psychology \& Health, vol. 27, no. 11, pp. 1323-1341, 2012.

[63] P. B. Parikh, J. Yang, S. Leigh et al., "The impact of financial barriers on access to care, quality of care and vascular morbidity among patients with diabetes and coronary heart disease," Journal of General Internal Medicine, vol. 29, no. 1, pp. 76-81, 2014.

[64] A. F. Chou, S. L. Stewart, R. C. Wild, and J. R. Bloom, "Social support and survival in young women with breast carcinoma," Psycho-Oncology, vol. 21, no. 2, pp. 125-133, 2012.

[65] D. Baucom, J. Kirby, and N. Pukay-Martin, "Men's psychological functioning in the context of women's breast cancer," Journal of Marital and Family Therapy, vol. 38, 2012.

[66] L. L. Northouse, M. C. Katapodi, L. Song, L. Zhang, and D. W. Mood, "Interventions with family caregivers of cancer patients meta-analysis of randomized trials," A Cancer Journal for Clinicians, vol. 60, pp. 317-339, 2010. 